Background Endovascular therapy (EVT) has become the first line treatment strategy for patients with large vessel occlusion strokes (LVOS). However, often more than one thrombectomy maneuver - either using stent-retrievers, thrombus aspiration or combined approaches - is necessary to achieve reperfusion. A true first pass effect with improved functional outcomes after MT has been described previously in single center data. Aim of the present study is to investigate the effect of true first-pass complete recanalization vs. multi-pass complete recanalization on functional outcome in a large, multicenter stroke database.

Methods Patients who underwent MT for LVOS in the anterior cerebral circulation between January 2014 to January 2021 and achieved complete reperfusion were identified from the Stroke Thrombectomy and Aneurysm Registry (STAR). We compared functional outcomes of patients with first- (defined as mTICI 3 after a single thrombectomy maneuver) vs. multiple-pass complete reperfusion (defined as mTICI 3 after $\geq 1$ thrombectomy maneuvers).

Results A total of 1481 patients with anterior circulation LVOS and successful recanalization were included in the analysis. First-pass complete recanalization was achieved in 778 patients vs. 703 patients with multi-pass complete reperfusion. Patients with first pass complete recanalization had lower NIHSS at baseline $(15 \pm 7$ vs $16 \pm 7$, $\mathrm{p}=0.056)$, had higher ASPECTS at baseline $(8.3$ vs 7.8 , $\mathrm{p}<0.001)$, were less likely to be male $(47 \%$ vs $51 \%$, $\mathrm{p}=0.078)$ and to have intracranial ICA occlusions $(14 \%$ vs. $27 \%)$ as well as more likely to have M1/M2 occlusions (86\% vs. $73 \%, \mathrm{o}<0.001)$, diabetes mellitus $(28 \%$ vs. $24 \%$, $\mathrm{p}=0.076)$, atrial fibrillation $(37 \%$ vs. $32 \%, \mathrm{p}=0.064)$. Patients with first pass complete reperfusion hat a trend towards a lower discharge NIHSS (8 vs. 10, $\mathrm{p}=0.075$ ) and higher improvement of the NIHSS (7 vs. 5, p=0.168). Among the factors lower age (OR 0.966, p<0.010), lower pre-stroke mRS (OR 0.601, p<0.001), diabetes mellitus (OR 0.612, $\mathrm{p}=0.014$ ), higher ASPECTS (OR 1.183, $\mathrm{p}<0.001)$ first pass complete recanalization persisted as a independent predictor of favorable functional outcome (defined as modified Rankin Scale $\leq 2$; OR 1.49, p=0.026).

Conclusion This analysis of a large, multi-center stroke database confirms the importance of first pass reperfusion in endovascular stroke care.

Disclosures I. Maier: None. M. Psychogios: None. J. Liman: None. S. Al Kasab: None. E. Almallouhi: None. A. Alawieh: None. S. Wolfe: None. K. Fargen: None. A. Arthur: None. N. Goyal: None. T. Dumont: None. P. Kan: None. J. Kin: None. R. De Leacy: None. J. Osbun: None. A. Rai: None. P. Jabbour: None. J. Grossberg: None. M. Park: None. R. Starke: None. R. Crosa: None. A. Spiotta: None. D. Behme: None.

\section{E-068 TOWARDS AUTOMATED INTRACRANIAL ANEURYSMAL BLEB DETECTION}

${ }^{1} \mathrm{D}$ Behme*, ${ }^{2} \mathrm{~L}$ Langolf, ${ }^{1} \mathrm{M}$ Thormann, ${ }^{1} \mathrm{~A}$ Mpotsaris, ${ }^{3} \mathrm{~B}$ Preim, ${ }^{4} \mathrm{P}$ Berg, ${ }^{5} \mathrm{~S}$ Saalfeld. ${ }^{1}$ Neuroradiology, University Clinic Magdeburg, Magdeburg, Germany; ${ }^{2}$ Research Campus Stimulate, University Magdeburg, Magdeburg, Germany; ${ }^{3}$ Department of Simulation and Graphics, University Magdeburg, Magdeburg, Germany; ${ }^{4}$ Research Campus Stimulate; Department of Fluid Mechanics and Technical Flows, University Magdeburg, Magdeburg, Germany; ${ }^{5}$ Research Campus Stimulate; Department of Simulation and Graphics, University Magdeburg, Magdeburg, Germany

\subsection{6/neurintsurg-2021-SNIS.163}

Introduction/Purpose Blebs of intracranial aneurysms are clear bulges or outpouchings of the aneurysms wall. Since irregularly shaped aneurysms, i.e. aneurysms with lobulated shapes including one or multiple blebs are associated with a higher rupture risk, bleb occurrence is important for

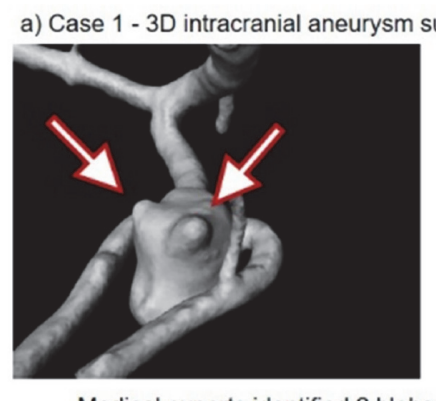

Medical experts identified 2 blebs

c) Case 2 - 3D intracranial aneurysm surface model

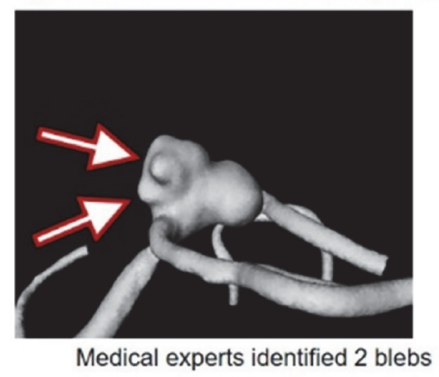

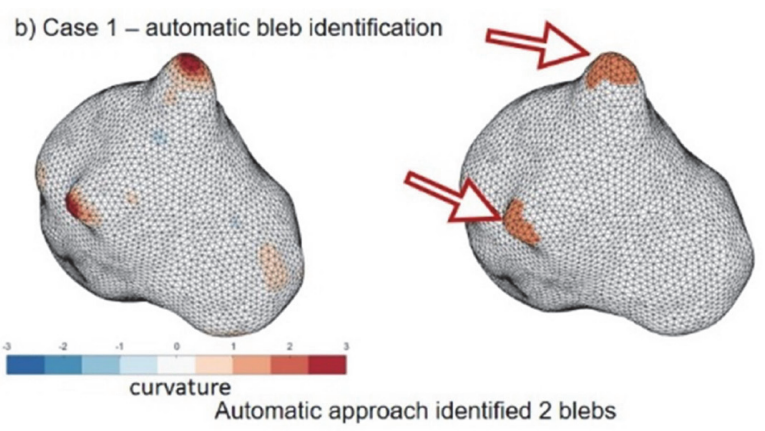

d) Case 2 - automatic bleb identification
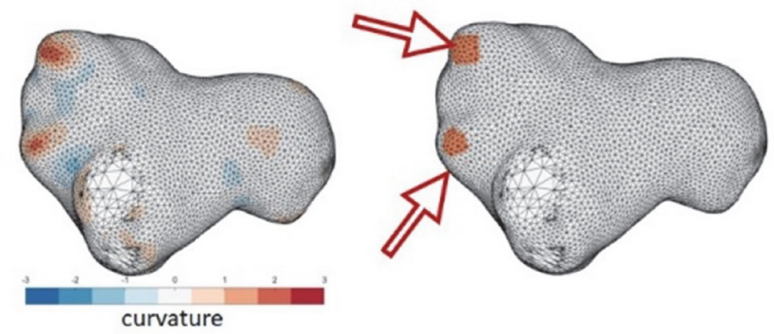

Automatic approach identified 2 blebs 
aneurysm treatment planning. Hence, an automatic computer-based extraction would be beneficial for bleb detection. On the contrary, a universal bleb definition is still missing thus a ground truth for bleb identification is required.

Materials and Methods We conducted an online survey with 40 intracranial aneurysm cases where clinical experts should state whether the intracranial aneurysm possesses none or multiple blebs. In addition, we developed an algorithm to automatically define blebs on the segmented 3D aneurysm surfaces. The algorithm extracts the Gaussian curvature for each vertex of the $3 \mathrm{D}$ aneurysm surface mesh, and divides the surface into regions with positive and negative curvature. Blebs were identified as regions with positive curvature, accompanied by areas of negative curvature.

Results A comparative analysis of our results reveals a strong variation between different raters which further strengthens the need for a bleb definition. The curvature-based automatic detection approach yields two advantages: First, the detection whether a bleb is present or not can support the medical expert when analyzing the aneurysm shape. Second, especially for multiple blebs, the ratings of the medical expert strongly varies in contrast to the automatic bleb detection approach. Hence, a computer-support provides a quantitative and reproducible result.

Conclusion Bleb definition is highly rater-dependent and standardized guidelines are required. Our approach employs curvature of the 3D intracranial aneurysm surfaces for automatic bleb identification to support the clinicians by highlighting lobulated shapes of the aneurysm itself and to reduce interobserver variability between medical experts.

Disclosures D. Behme: None. L. Langolf: None. M. Thormann: None. A. Mpotsaris: None. B. Preim: None. P. Berg: None. S. Saalfeld: None.

\section{E-069 DISCRIMINATION BETWEEN STABLE AND UNSTABLE INTRACRANIAL ANEURYSMS USING IMAGE-BASED BLOOD FLOW SIMULATIONS}

${ }^{1} \mathrm{D}$ Behme*, ${ }^{2} \mathrm{~S}$ Voß, ${ }^{3} \mathrm{~J}$ Korte, ${ }^{1} \mathrm{M}$ Thormann, ${ }^{1} \mathrm{~A}$ Mpotsaris, ${ }^{4} \mathrm{~S}$ Saalfeld, ${ }^{5} \mathrm{G}$ Janiga, ${ }^{3} \mathrm{P}$ Berg. ${ }^{1}$ Neuroradiology, University Clinic Magdeburg, Magdeburg, Germany; ${ }^{2}$ Research Campus Stimulate, University Magdeburg, Magdeburg, Germany; ${ }^{3}$ Research Campus Stimulate; Department of Fluid Mechanics and Technical Flows, University Magdeburg, Magdeburg, Germany; ${ }^{4}$ Research Campus Stimulate; Department of Simulation and Graphics, University Magdeburg, Magdeburg, Germany; ${ }^{5}$ Department of Fluid Dynamics and Technical Flows, University Magdeburg, Magdeburg, Germany

\subsection{6/neurintsurg-2021-SNIS.164}

Introduction/Purpose The discrimination between hemodynamically stable and unstable intracranial aneurysms remains challenging. Recent studies reveal the potential existence of intraaneurysmal velocity fluctuations, which appear at high frequency. Since these flow disturbances might promote the vessel wall remodeling process, indications regarding the occurrence of such phenomena are desired.

Materials and Methods To quantify unstable hemodynamics, image-based blood flow simulations were carried out in a ruptured aneurysm at the posterior inferior cerebellar artery.

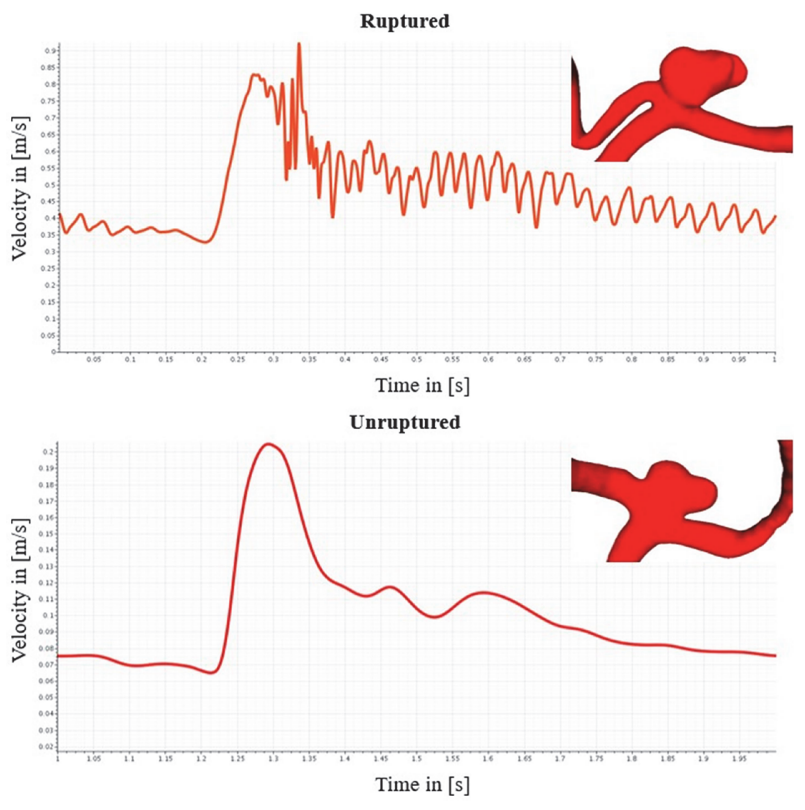

Abstract E-069 Figure 1 Intra-aneurysmal velocity waveform for the ruptured (top) and reference unruptured (bottom) intracranial aneurysm. Notice the high-frequency fluctuations throughout the whole cardiac cycle for the ruptured case

Since the exact rupture site could be identified during the angiographic imaging, local flow analysis was feasible. Beside the evaluation of time-varying velocity fluctuations, shear stress distributions and vortex generations were analyzed. Furthermore, the existing spectral flow entropy was assessed using proper orthogonal decomposition. Finally, all simulations were repeated in an unruptured aneurysm at the middle cerebral artery, which was similar with respect to size and shape.

Results The high-resolution blood flow simulations reveal the existence of complex flow patterns in combination with highfrequency fluctuations. These predominantly occur after the peak-systolic inflow and maintain in the diastolic phase. Furthermore, the wall shear stress distribution demonstrates a strong oscillatory behavior emphasizing the unstable character of the flow. The calculation of the spectral entropy resulted in a value of 0.76 , which was associated with highly disturbed flow in advance. In contrast, these observations are absent in the reference simulation of the unruptured aneurysm. Specifically, stable flow patterns are visible and a low spectral entropy of 0.12 was calculated (a value of 0 represents steady flow conditions).

Conclusion The presence of high-frequency fluctuations and increased spectral entropy could be a potential biomarker for the discrimination between hemodynamically stable and unstable intracranial aneurysms. Since strong deviations were detectable in aneurysms with similar shape and location, stronger focus on the surrounding vasculature (e.g., with respect to the angle of proximal and distal branches) is suggested.

Disclosures D. Behme: None. S. Voß: None. J. Korte: None. M. Thormann: None. A. Mpotsaris: None. S. Saalfeld: None. G. Janiga: None. P. Berg: None. 